\title{
Photoacoustic Imaging of Tattoo Inks: Phantom and Clinical Evaluation
}

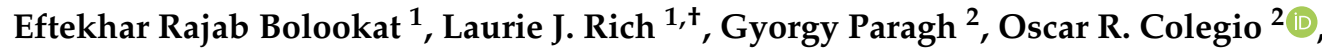 \\ Anurag K. Singh ${ }^{3}$ and Mukund Seshadri ${ }^{1,4, *}$
}

1 Laboratory for Translational Imaging, Center for Oral Oncology, Roswell Park Comprehensive Cancer Center, Buffalo, NY 14263, USA; Eftekhar.RajabBolookat@roswellpark.org (E.R.B.);

Laurie.Rich@pennmedicine.upenn.edu (L.J.R.)

2 Department of Dermatology, Roswell Park Comprehensive Cancer Center, Buffalo, NY 14263, USA; Gyorgy.Paragh@roswellpark.org (G.P.); Oscar.Colegio@roswellpark.org (O.R.C.)

3 Department of Radiation Medicine, Roswell Park Comprehensive Cancer Center, Buffalo, NY 14263, USA; Anurag.Singh@roswellpark.org

4 Department of Dentistry and Maxillofacial Prosthetics, Roswell Park Comprehensive Cancer Center, Buffalo, NY 14263, USA

* Correspondence: Mukund.Seshadri@roswellpark.org; Tel.: +1-716-845-1552

+ Present affiliation: Center for Magnetic Resonance and Optical Imaging, Department of Radiology, University of Pennsylvania, PA 19104, USA.

Received: 3 January 2020; Accepted: 31 January 2020; Published: 4 February 2020

\begin{abstract}
Photoacoustic imaging (PAI) is a novel hybrid imaging modality that provides excellent optical contrast with the spatial resolution of ultrasound in vivo. The method is widely being investigated in the clinical setting for diagnostic applications in dermatology. In this report, we illustrate the utility of PAI as a non-invasive tool for imaging tattoos. Ten different samples of commercially available tattoo inks were examined for their optoacoustic properties in vitro. In vivo PAI of an intradermal tattoo on the wrist was performed in a healthy human volunteer. Black/gray, green, violet, and blue colored pigments provided higher levels of PA signal compared to white, orange, red, and yellow pigments in vitro. PAI provided excellent contrast and enabled accurate delineation of the extent of the tattoo in the dermis. Our results reveal the photoacoustic properties of tattoo inks and demonstrate the potential clinical utility of PAI for intradermal imaging of tattoos. PAI may be useful as a clinical adjunct for objective preoperative evaluation of tattoos and potentially to guide/monitor laser-based tattoo removal procedures.
\end{abstract}

Keywords: photoacoustic imaging; tattoo; dermatology; ultrasound

\section{Introduction}

Non-invasive in vivo imaging has been an integral part of the armamentarium in dermatology for decades $[1,2]$. In addition to traditional dermoscopy and ultrasound (US), optical imaging methods such as Raman spectroscopy, confocal microscopy, and optical coherence tomography have demonstrated utility in the diagnosis of skin diseases [3-5]. Photoacoustic imaging (PAI) or optoacoustic imaging is a relatively new imaging method that provides excellent optical contrast with the spatial resolution of US [6,7]. Although administration of exogenous agents can enhance contrast on PAI, this is not essential given the abundance of endogenous chromophores such as melanin and hemoglobin in tissue. Clinical studies have demonstrated the usefulness of PAI as a label-free imaging tool for assessment of melanomas and non-melanoma skin cancers [8-10] and to visualize microvascular and inflammatory changes in the skin [11]. However, the ability of PAI to visualize tattoos has not been previously reported. Since tattoo dyes are known to absorb and reflect light, we hypothesized that 
PAI can visualize and determine the three-dimensional localization of tattoo pigments. To test this hypothesis, we first examined the optoacoustic properties of commercially available tattoo inks in a tissue-mimicking phantom. Subsequently, we examined the ability of PAI to visualize an intradermal tattoo in a healthy volunteer.

\section{Materials and Methods}

In vitro examination of the photoacoustic properties of the inks was performed using hollow channels created within a tissue-mimicking phantom composed of agarose (Bio Rad, Hercules, CA, USA) and intralipid (Sigma, St. Louis, MO, USA). The synthesis of the phantom has been previously described [12]. Ten samples of commercially available (Scream Ink; Worldwide Tattoo Supply) tattoo inks (black, pitch black, blue, gray, green, violet, orange, red, yellow, and white) (Figure 1a) were used. The samples were diluted in phosphate buffered saline (1:1) and injected into the channels. Whole blood andphosphate-buffered saline (PBS) were also injected into separate channels for comparison (Figure 1b). The PA images were obtained using a $21 \mathrm{MHz}$ probe with the following settings: 2D multi-wavelength PA mode: 680-900 nm; Gain: 40 dB, Depth: 20.00 mm, Width: 23.04 mm, Persistence: 3.

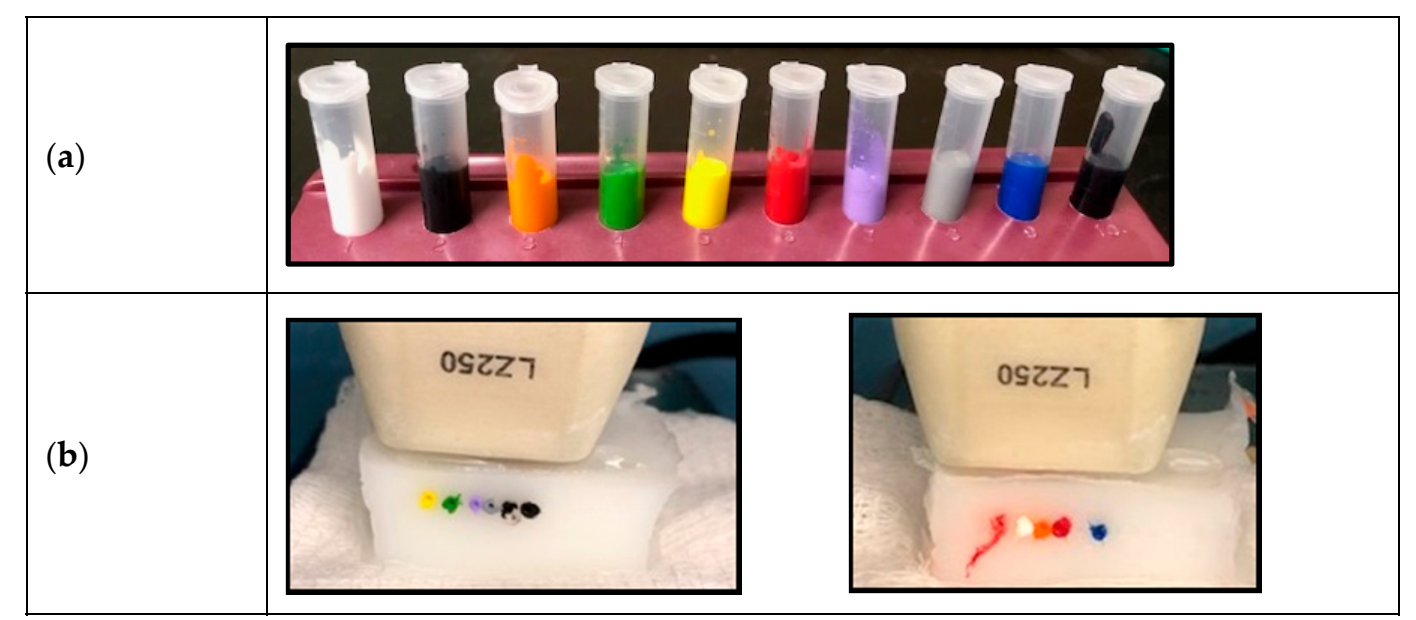

Figure 1. (a) Photograph showing the ten commercially available tattoo ink samples evaluated in vitro. (b) Set up for evaluation of photoacoustic properties of tattoo inks using hollow channels created in a tissue mimicking phantom.

An institutional review board (IRB Protocol \#48917) approved pilot clinical study was conducted at Roswell Park Comprehensive Cancer Center. A healthy volunteer with an existing tattoo on the wrist was enrolled to participate in the imaging study. Written informed consent was obtained prior to imaging examination. Prior to image acquisition, the volunteer was comfortably seated, and acoustic gel applied to the skin overlying the tattoo. Combined B-mode US and PAI of the tattoo on the left wrist in the healthy volunteer was performed using a commercially available $21 \mathrm{MHz}$ linear-array transducer system (bandwidth of 13-24 MHz; axial resolution $75 \mu \mathrm{m}$, lateral resolution $165 \mu \mathrm{m}$ imaging; maximum FOV $23 \times 30 \mathrm{~mm}$, focal zone of $15 \mathrm{~mm}$ (Vevo ${ }^{\circledR}$ LAZR; VisualSonics Inc., Toronto). For ease of visualization, pseudo colorized PA maps were generated. Contrast (50) and brightness (90) levels were set prior to application of the color map on the images. The tattoo on the left wrist of the volunteer was examined in the transverse and longitudinal planes. The transducer was gently placed on the skin surface and US-PAI images were obtained. The imaging procedure took approximately $15 \mathrm{~min}$. Following completion of imaging, imaging datasets were transferred to the imaging workstation for offline processing (Vevo LAB 3.2.0, VisualSonics, Toronto, Canada, 2019). 


\section{Results}

Among the tattoo inks tested, black/gray, green, violet, and blue colored pigments provided higher levels of PA signal (Figure $2 \mathrm{a}-\mathrm{c}$ ) in vitro. Yellow, red, and orange had a comparable signal to blood and white had the lowest signal detected by PAI (Figure 2a,d). In the volunteer, the skin overlying the tattoo appeared normal without any evidence of inflammatory change on clinical examination (Figure 3). In vivo PAI provided excellent contrast and enabled accurate delineation of the extent of the tattoo in the dermis. The reconstructed MIP image allowed for visualization of the tattoo in the dermis in an unambiguous manner. PAI allowed for visualization of the tattoo in the pigmented skin and the surrounding region (Figure 3).

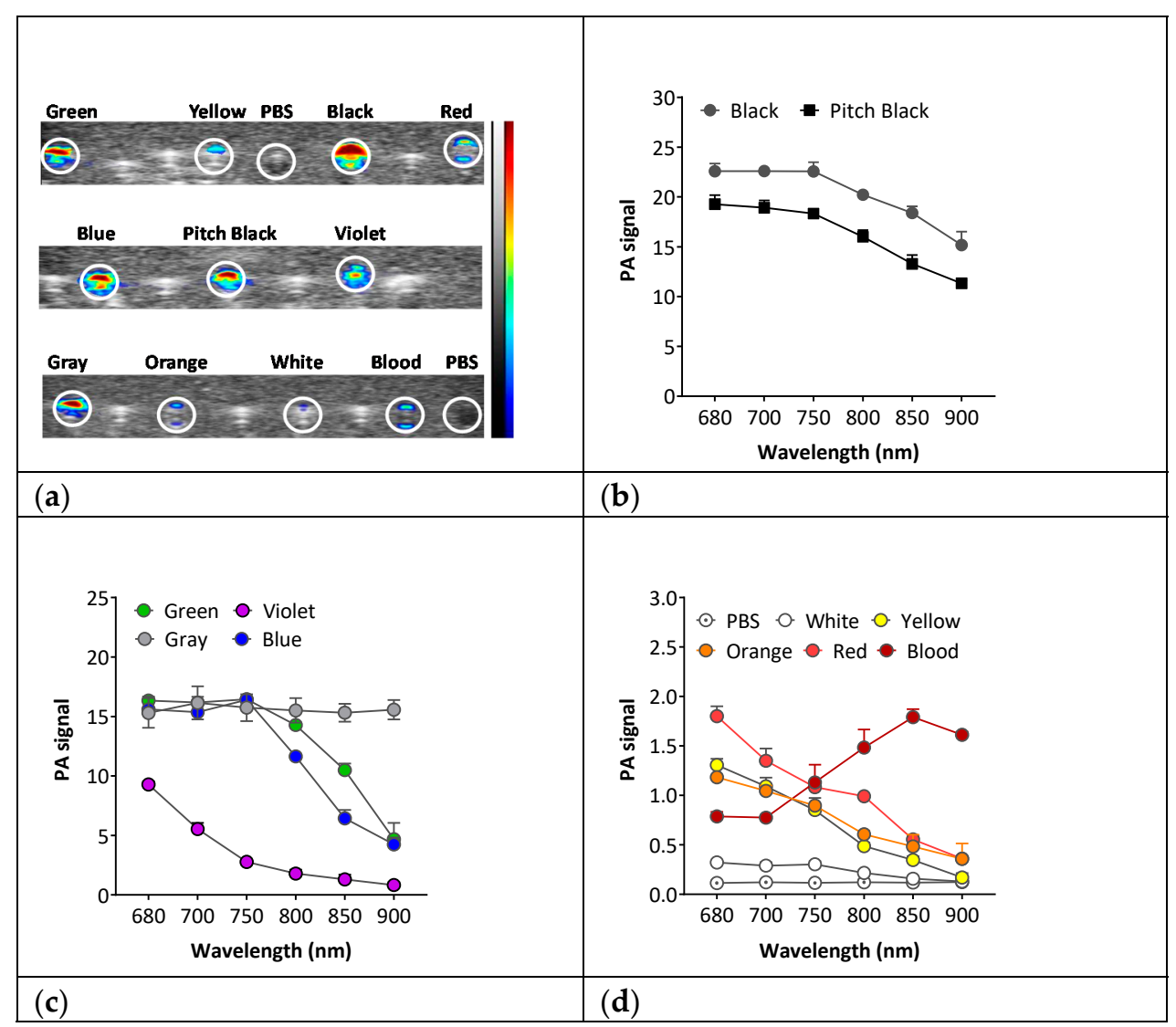

Figure 2. PAI of tattoo inks in vitro. (a) Pseudo-colorized PA signal maps $(680 \mathrm{~nm})$ of the ten tattoo inks along with blood and PBS filled channels. (b-d) Plots showing PA signal from 680-900 nm from the ten tattoo ink samples along with blood and PBS. 


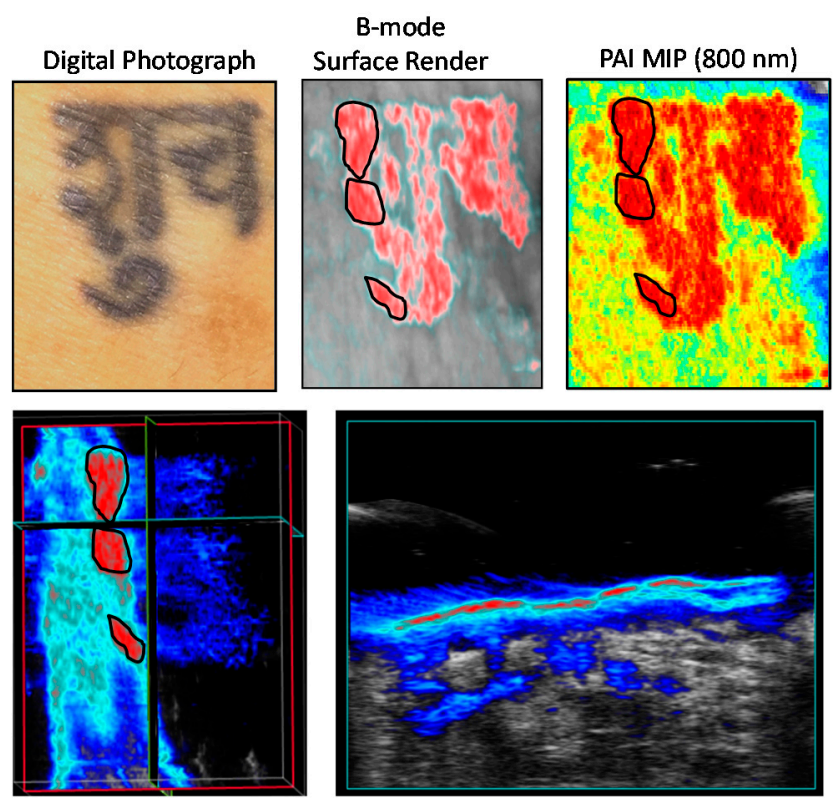

Figure 3. PAI of an intradermal tattoo. The panel of images on the top represents digital photograph (left), a B-mode surface rendering (middle), and a reconstructed maximum intensity projection (MIP; right) image $(800 \mathrm{~nm})$ of a black tattoo on the wrist of a healthy volunteer. Corresponding longitudinal (left) and transverse axial (right) images of a part of the tattoo (outlined in black) is shown. The images have been pseudo-colorized for enhanced visualization.

\section{Discussion}

In this report, we used PAI a relatively new optical imaging method for imaging tattoo inks in vitro and for in vivo visualization of a tattoo in the wrist of a healthy volunteer. Given its portability, low cost and high resolution, US is widely used in dermatology [13]. Clinical studies have also demonstrated the usefulness of US for imaging musculoskeletal anatomy [14,15]. Tattoo inks exhibited strong yet distinct photoacoustic properties that can be exploited for non-invasive visualization using PAI. Our phantom study demonstrated that the PA signal varies greatly among tattoo inks. Lighter colors like white mostly reflect laser light so there is less absorption and low PA signal. Darker tattoo inks, especially the black color generates more robust signals. Dermal tattoo pigmentation was successfully imaged in vivo using PAI. Our study demonstrates the feasibility of PAI to spatially map tattoos in human skin. To the best of our knowledge, this is the first report on the use of PAI for visualization of an intradermal tattoo.

While tattooing is relatively cheap, tattoo removal is an expensive procedure that involves use of laser light and relies on photolysis of the tattoo pigment. Currently, laser removal of tattoos including the wavelength of the laser light and fluence is based on qualitative, visual examination. As a result, the outcome following tattoo removal procedures is dependent on the experience of the clinician. A non-invasive imaging method that can provide an objective assessment of the extent of the tattoo in all three dimensions could be valuable in guiding tattoo removal. In this regard, PAI could serve as a relatively inexpensive, non-invasive, clinical tool for preoperative evaluation of tattoos and for objective monitoring of laser removal procedures.

Author Contributions: Conceptualization: M.S., A.K.S., G.P.; Data curation: E.R.B., L.J.R., A.K.S., M.S.; Formal analysis: E.R.B., L.J.R., M.S.; Funding acquisition: A.K.S., M.S.; Investigation: E.R.B., L.J.R., A.K.S., M.S.; Methodology: E.R.B., L.J.R., A.K.S., M.S.; Project administration: A.K.S., M.S.; Resources: A.K.S., M.S.; Supervision: A.K.S., M.S.; Validation: E.R.B., L.J.R., G.P., O.R.C., A.K.S., M.S.; Visualization: E.R.B., L.J.R., O.R.C., M.S.; Writing—original draft preparation: E.R.B., L.J.R., M.S.; Writing—review and editing: E.R.B., L.J.R., G.P., O.R.C., A.K.S., M.S. All authors have read and agreed to the published version of the manuscript.

Funding: This work was supported by grants from the National Cancer Institute 1R01CA204636, National Center for Research Resources S10OD010393-01 and the Alliance Foundation of Western New York (to MS), and utilized 
shared resources supported by Roswell Park Cancer Institute Cancer Center Support Grant from the National Cancer Institute P30CA06156. The funding sponsors had no role in the design of the study, collection, analyses, or interpretation of data, writing of the manuscript, and in the decision to publish the results.

Conflicts of Interest: The authors declare no conflict of interest.

\section{References}

1. Hamblin, M.R.; Avci, P.; Gupta, G.K. Imaging in Dermatology, 1st ed.; Academic Press: London, UK, 2016.

2. Hibler, B.P.; Qi, Q.; Rossi, A.M. Current state of imaging in dermatology. Semin. Cutan. Med. Surg. 2016, 1, 2-8. [CrossRef] [PubMed]

3. Patil, C.A.; Kirshnamoorthi, H.; Ellis, D.L.; van Leeuwen, T.G.; Mahadevan-Jansen, A. A Clinical Instrument for Combined Raman Spectroscopy-Optical Coherence Tomography of Skin Cancers. Lasers Surg. Med. 2011, 2, 143-151. [CrossRef] [PubMed]

4. Dubois, A.; Levecq, O.; Azimani, H.; Siret, D.; Barut, A.; Suppa, M.; Del Marmol, V.; Malvehy, J.; Cinotti, E.; Rubegni, P.; et al. Line-field confocal optical coherence tomography for high-resolution noninvasive imaging of skin tumors. J. Biomed. Opt. 2018, 10, 1-9. [CrossRef] [PubMed]

5. Tkaczyk, E.R. Innovations and developments in dermatologic non-invasive optical imaging and potential clinical applications. Acta derm Venereol. 2017, 97 (Suppl. S218), 5-13. [CrossRef] [PubMed]

6. Kruger, R.A. Photoacoustic ultrasound. Med. Phys. 1994, 21, 127-131. [CrossRef] [PubMed]

7. Wang, L.V. Ultrasound-mediated biophotonic imaging: A review of acousto-optical tomography and photo-acoustic tomography. Dis. Markers. 2004, 19, 123-138. [CrossRef] [PubMed]

8. Zeitouni, N.C.; Rohrbach, D.J.; Aksahin, M.; Sunar, U. Preoperative ultrasound and photoacoustic imaging of nonmelanoma skin cancers. Dermatol. Surg. 2015, 41, 525-528. [CrossRef] [PubMed]

9. Schwarz, M.; Buehler, A.; Aguirre, J.; Ntziachristos, V. Three-dimensional multispectral optoacoustic mesoscopy reveals melanin and blood oxygenation in human skin in vivo. J. Biophotonics 2016, 9, 55-60. [CrossRef] [PubMed]

10. Zhou, Y.; Tripathi, S.V.; Rosman, I.; Ma, J.; Hai, P.; Linette, G.P.; Council, M.L.; Fields, R.C.; Wang, L.V.; Cornelius, L.A. Noninvasive Determination of Melanoma Depth using a Handheld Photoacoustic Probe. J. Investig. Dermatol. 2017, 137, 1370-1372. [CrossRef] [PubMed]

11. Hindelang, B.; Aguirre, J.; Schwarz, M.; Berezhnoi, A.; Eyerich, K.; Ntziachristos, V.; Biedermann, T.; Darsow, U. Non-invasive imaging in dermatology and the unique potential of raster-scan optoacoustic mesoscopy. J. Eur. Acad. Dermatol. Venereol. 2019, 33, 1051-1061. [CrossRef] [PubMed]

12. Rich, L.; Seshadri, M. Photoacoustic Imaging of Vascular Hemodynamics: Validation with Blood Oxygenation Level-Dependent MR imaging. Radiology 2015, 275, 110-118. [CrossRef] [PubMed]

13. Kleinerman, R.; Whang, T.B.; Bard, R.L.; Marmur, E.S. Ultrasound in dermatology: Principles and applications. J. Am. Acad. Dermatol. 2012, 67, 478-487. [CrossRef] [PubMed]

14. Wu, W.T.; Chang, K.V.; Mezian, K.; Naňka, O.; Lin, C.P.; Özçakar, L. Basis of shoulder nerve entrapment syndrome: An ultrasonographic study exploring factors influencing cross-sectional area of the suprascapular nerve. Front. Neurol. 2018, 9, 902. [CrossRef] [PubMed]

15. Chang, K.V.; Yang, K.C.; Wu, W.T.; Huang, K.C.; Han, D.S. Association between metabolic syndrome and limb muscle quantity and quality in older adults: A pilot ultrasound study. Diabetes Metab. Syndr Obes. 2019, 12, 1821-1830. [CrossRef] [PubMed]

(C) 2020 by the authors. Licensee MDPI, Basel, Switzerland. This article is an open access article distributed under the terms and conditions of the Creative Commons Attribution (CC BY) license (http://creativecommons.org/licenses/by/4.0/). 